\title{
PROPERTIES OF MINIMIZERS OF AVERAGE-DISTANCE PROBLEM VIA DISCRETE APPROXIMATION OF MEASURES
}

\author{
XIN YANG LU AND DEJAN SLEPČEV
}

ABSTRACT. Given a finite measure $\mu$ with compact support, and $\lambda>0$, the averagedistance problem, in the penalized formulation, is to minimize

$$
E_{\mu}^{\lambda}(\Sigma):=\int_{\mathbb{R}^{d}} d(x, \Sigma) d \mu(x)+\lambda \mathcal{H}^{1}(\Sigma)
$$

among pathwise connected, closed sets, $\Sigma$. Here $d(x, \Sigma)$ is the distance from a point to a set and $\mathcal{H}^{1}$ is the 1-Hausdorff measure. In a sense the problem is to find a onedimensional measure that best approximates $\mu$. It is known that the minimizer $\Sigma$ is topologically a tree whose branches are rectifiable curves. The branches may not be $C^{1}$, even for measures $\mu$ with smooth density. Here we show a result on weak second-order regularity of branches. Namely we show that arc-length-parameterized branches have $B V$ derivatives and provide a priori estimates on the $B V$ norm.

The technique we use is to approximate the measure $\mu$, in the weak- $*$ topology of measures, by discrete measures. Such approximation is also relevant for numerical computations. We prove the stability of the minimizers in appropriate spaces and also compare the topologies of the minimizers corresponding to the approximations with the minimizer corresponding to $\mu$.

Keywords. nonlocal variational problem, average-distance problem, regularity Classification. 49Q20, 49K10, 49Q10, 35B65

\section{INTRODUCTION}

The average-distance problem was introduced by Buttazzo, Oudet and Stepanov in [1]. In the penalized formulation the problem takes the form

Problem 1.1. For $d \geq 2$, given a finite compactly supported measure $\mu$, and $\lambda>0$, minimize

$$
E_{\mu}^{\lambda}(\Sigma)=\int_{\mathbb{R}^{d}} d(x, \Sigma) d \mu(x)+\lambda \mathcal{H}^{1}(\Sigma)
$$

among pathwise connected, closed sets $\Sigma$.

We define the set of admissible candidates by

$$
\mathcal{A}:=\left\{\Sigma \subseteq \mathbb{R}^{d}: \Sigma \text { compact, pathwise connected, and } \mathcal{H}^{1}(\Sigma)<\infty\right\} .
$$

Date: January 8, 2013. 
For the future reference we denote the part of the energy functional which measures the approximation error by

$$
F_{\mu}(\Sigma):=\int_{\mathbb{R}^{d}} d(x, \Sigma) d \mu .
$$

This problem has many applications: a simple one is

- $\mu$ is the distribution of passengers,

- $\Sigma$ is a transport network.

In this case $F_{\mu}(\Sigma)$ is the average distance of passengers from the network, and $\mathcal{H}^{1}(\Sigma)$ is the cost to build such network. Minimizing $E_{\mu}^{\lambda}$ is determining the network with best serves the passengers, under cost considerations.

An alternative interpretation is found in cloud data systems:

- $\mu$ is the distribution of data points,

- $\Sigma$ is a one dimension object which approximates the data.

In this case $F_{\mu}(\Sigma)$ is the approximation error, while $\lambda \mathcal{H}^{1}(\Sigma)$ is the cost associated to the complexity of such representation. Minimizing $E_{\mu}^{\lambda}$ is determining the best approximation, accounting for the complexity.

Existence of minimizers, follows from theorems of Blaschke and Gołą, [1]. Geometric properties of minimizers were investigated by Buttazzo, Oudet and Stepanov [1], Buttazzo and Stepanov [2, 3] (mainly in two dimensions) and by Paolini and Stepanov [9] (for higher dimensions). Let us also add that Lemenant [6] provides an excellent review of the current knowledge about average-distance problem. It has been proven that minimizers are topologically trees with rectifiable branches. Furthermore no more than three branches can meet at a point. Regarding the regularity of branches the following results are available:

- In [1] it has been proven that minimizers are Ahlfors regular in two dimensional domains. The result was extended to higher dimensional domains in [9],

- In [2] it has been proven that minimizers are union of Lipschitz curves; for the penalized problem, it is easy to prove that this union if finite (e.g. using Theorem 3.4).

- In [12] it has been proven that regularity beyond $C^{1,1}$ cannot be expected in the general case.

- In [10] an explicit counterexample to $C^{1}$ regularity for minimizers has been constructed.

We recall that in two dimensional domains, in the constrained formulation

$$
\min _{\mathcal{H}^{1}(\Sigma) \leq L} F_{\mu}(\Sigma)
$$

in [11] it has been proven that given a minimizer $\Sigma_{\text {opt }}$, which is finite union of Lipschitz curves $\left\{\gamma_{i}\right\}_{i=1}^{N}$ (as proven in [2]), each such curve $\gamma_{j}$ is $C^{1,1}$ away from the points where a 
positive mass projects, at which corners are possible. Moreover, in [7] which studies the average-distance problem in two dimensions provides "tilt estimates" which are related to our regularity estimates.

This paper aims to extend such regularity results, and prove that minimizers are finite unions of Lipschitz curves with $B V$ derivatives. More precisely we show that given an arbitrary nonnegative finite measure with compact support, $\mu$, and $\lambda>0$, any solution $\Sigma \in \operatorname{argmin} E_{\mu}^{\lambda}$ is finite union of Lipschitz curves $\left\{\gamma_{k}\right\}_{k=1}^{N}$ (without loss of generality assume that all $\gamma_{k}$ are arc-length parameterized), such that for any $k=1, \ldots, N$ it holds

$$
\sum_{k}\left\|\gamma_{k}^{\prime}\right\|_{T V} \leq \frac{1}{\lambda}\left|\mu\left(\mathbb{R}^{d}\right)\right|,
$$

where $\|\cdot\|_{T V}$ denotes the total variation.

In other words we provide an estimate on the total curvature of the curves that make up $\Sigma$, where the curvature, $\kappa=\gamma_{k}^{\prime \prime}$ is understood as the signed measure. The fact the the total mass (times $1 / \lambda$ ) bounds the curvature is not surprising. To motivate it let us assume that the minimizer $\Sigma$ is a single smooth curve and that $\mu$ is absolutely continuous with respect to Lebesgue measure. Let $\Pi$ be the projection onto $\Sigma$ (it is known that $\Pi$ has unique value almost everywhere). Then the first variation for the Problem 1.1 gives that for any smooth vector field $v: \Sigma \rightarrow \mathbb{R}^{d}$ supported away from the endpoints of $\Sigma$

$$
-\int_{\Sigma} \kappa \cdot v d \mathcal{H}^{1}(\Sigma)=\frac{1}{\lambda} \int_{\mathbb{R}^{d}} \frac{(x-\Pi(x))}{|x-\Pi(x)|} \cdot v((\Pi(x)) d \mu(x) .
$$

Taking supremum over all $v$, as above, with $|v| \leq 1$ implies (1.2).

The difficulties one faces in applying the reasoning above are that $\Sigma$ is neither regular nor a curve and that $\mu$ is not assumed to be absolutely continuous with respect to the Lebesgue measure.

The approach used is based on approximating a measure $\mu$ by a sequence discrete measures $\left\{\mu_{n}\right\} \stackrel{*}{*} \mu$, and analyzing the minimizers of Problem 1.1 with $\mu$ replaced by $\mu_{n}$. In addition to the estimate on the BV norm we prove a topological relation between minimizers of $E_{\mu_{n}}^{\lambda}$ and minimizers of $E_{\mu}^{\lambda}$, with $\lambda>0$ arbitrarily given.

This paper is structured as follows:

- In Section 2 we recall the known results on the average-distance problem, introduce the discrete approximations and prove a couple of preliminary results.

- In Section 3 we prove an upper bound on the number endpoints of minimizers and analyze the behavior of endpoints in the approximation process. In particular we prove in Theorem 3.4 that if $\mu_{n}$ are discrete approximations of $\mu$ and $\Sigma_{n}$ are minimizers of $E_{\mu_{n}}^{\lambda}$ which converge to $\Sigma$, a minimizer of $E_{\mu}^{\lambda}$, then each endpoint of $\Sigma$ is a limit of endpoints of $\Sigma_{n}$. 
- Section 4 is devoted to the topological comparison between minimizers for the approximate problem and the minimizer corresponding to $\mu$.

- In Section 5 we prove prove that minimizers of the average-distance problem are finite union of Lipschitz curves with $B V$ derivatives, and prove a priori estimates on $B V$ norms in Theorem 5.1.

\section{PRELIMINARY RESULTS}

We restrict our attention to probability measures, $\mu$, purely for notational simplicity. Given a probability measure $\mu$ and a compact set $\Sigma$, we need to find a "projection" of the measure $\mu$ onto $\Sigma$. The issue is that for $x \in \mathbb{R}^{d}$, the minimizer of $|x-y|$ over $y \in \Sigma$ is in general not unique. In fact it has been proven by the authors in [8] that the ridge of $\Sigma$ (points having nonunique projection on $\Sigma$ ) is an $\mathcal{H}^{d-1}$-rectifiable set. If $\mu$ is absolutely continuous with respect to the Lebesgue measure, $\mu \ll \mathcal{L}^{d}$, then the ridge is $\mu$-negligible and thus the projection $\Pi: x \mapsto \operatorname{argmin}_{y \in \Sigma}|x-y|$ is unique $\mu$-a.e. and one can define the projection of $\mu$ onto $\Sigma$ by $\sigma=\Pi_{\sharp} \mu$ (the push-forward of the measure).

However if $\mu$ is not absolutely continuous with respect to Lebesgue measure then more care is needed.

Lemma 2.1. Let $\mu$ be a probability measure and $\Sigma$ a compact set. There exists a probability measure $\pi$ on $\mathbb{R}^{d} \times \Sigma$ such that the first marginal of $\pi$ is $\mu$ (that is $\pi\left(A \times \mathbb{R}^{d}\right)=\mu(A)$ for any Borel set $A$ ) and that for $\pi$-a.e. $(x, y),|x-y|=\min _{z \in \Sigma}|x-z|$.

We define $\sigma$, the projection of $\mu$ onto $\Sigma$, to be the second marginal of $\pi$. While $\pi$ is in general not unique, from the outset we choose one $\pi$ and the corresponding $\sigma$. All statements we make hold for any $\pi$ chosen.

Proof. Let $\mathcal{P}_{\Sigma}$ be the set of Borel probability measures on $\Sigma$. Consider the functional $\sigma \mapsto d_{W}(\mu, \sigma)$ on $\mathcal{P}_{\Sigma}$, where $d_{W}$ is the Wasserstein distance. Since $\Sigma$ is compact, $\mathcal{P}_{\Sigma}$ is sequentially compact with respect to weak-* convergence of measures. Given that $d_{W}(\mu, \cdot)$ is continuous on $\mathcal{P}_{\Sigma}$ with respect to the weak-* convergence of measures we conclude that there exists $\bar{\sigma} \in \mathcal{P}_{\Sigma}$ minimizing the Wasserstein distance to $\mu$. Let $\pi$ be the optimal transportation plan (for the quadratic cost) between $\mu$ and $\bar{\sigma}$. We claim that $\pi$ has the desired properties.

Since, by definition, the first marginal of $\pi$ is $\mu$ we only need to verify that for $\pi$-a.e. $(x, y),|x-y|=\min _{z \in \Sigma}|x-z|$. Assume that this is not the case, that is that there exists $(x, y) \in \operatorname{supp} \pi$ and $z \in \Sigma$ such that $|x-y|>|x-z|$. Let $\delta=(|x-y|-|x-z|) / 3$ and $U=B(x, \delta) \times(B(y, \delta) \cap \Sigma)$. Since $(x, y) \in \operatorname{supp} \pi, \varepsilon:=\pi(U)>0$. Let $\pi_{\text {new }}=\pi-\left.\pi\right|_{U}+\varepsilon \delta_{(x, z)}$ and let $\sigma_{\text {new }}$ the the second marginal of $\pi_{\text {new }}$. Then $\sigma_{\text {new }} \in \mathcal{P}_{\Sigma}$ and $d_{W}\left(\mu, \sigma_{\text {new }}\right)<d_{W}(\mu, \bar{\sigma})$ which contradicts the fact that $\bar{\sigma}$ is a minimizer.

We introduce some notation and terminology: 
- The measure $\sigma$ defined after the statement of Lemma 2.1 can have atoms. For simplicity for $y \in \Sigma$ we write $\sigma(y)$ to mean $\sigma(\{y\})$.

- Sometimes it is important to emphasize which $\mu$ and $\Sigma$ the measure $\sigma$ corresponds to. Then we write $\sigma(\mu, \Sigma, A)$ for $\sigma(\mathcal{A})$, where $A$ is a measurable subset of $\Sigma$.

- The order of a point $y \in \Sigma$ is defined to be the number of pathwise connected components of $\Sigma \backslash\{y\}$. As we mentioned earlier if $\Sigma$ is a minimizer of $E_{\mu}^{\lambda}$ then all points on $\Sigma$ are of order 1,2, or 3 .

- Point $y \in \Sigma$ of order one is called an endpoint. We show in Lemma 3.1 that for any endpoint $\sigma(\mu, \Sigma, y) \geq \lambda$. We denote the set of endpoints of $\Sigma$ by $\operatorname{ext}(\Sigma)$. A point of order three is called a triple junction. A point $y \in \Sigma$ is called a corner if it is of order two and $\sigma(\mu, \Sigma, y)>0$.

We note that there is a simple bound on the length of the minimizers of $E_{\mu}^{\lambda}$. Namely if $\Sigma$ is a minimizer of $E_{\mu}^{\lambda}$ then

$$
\mathcal{H}^{1}(\Sigma) \leq \frac{1}{\lambda} \operatorname{diam} \operatorname{supp}(\mu) .
$$

The reason is that for any $z \in \operatorname{supp} \mu$ the minimality of $\Sigma$ implies

$$
\begin{aligned}
\lambda \mathcal{H}^{1}(\Sigma) \leq E_{\mu}^{\lambda}(\Sigma) & \leq E_{\mu}^{\lambda}(\{z\}) \\
& =\int_{\mathbb{R}^{d}} d(y,\{z\}) d \mu(y)=\int_{\operatorname{supp}(\mu)} d(y,\{z\}) d \mu(y) \leq \operatorname{diam} \operatorname{supp}(\mu) .
\end{aligned}
$$

We recall the following facts on the average-distance problem. We refer to [10] for further details.

(i) For any probability measure $\mu$ on $\mathbb{R}^{d}$, and $\lambda>0$, the functional $E_{\mu}^{\lambda}$ is lower semicontinuous w.r.t. $d_{\mathcal{H}}$.

(ii) Given $\Sigma \in \mathcal{A}$, and $\lambda>0$, the mapping $\Sigma \mapsto E_{\mu}^{\lambda}(\Sigma)$ is continuous w.r.t. weak-* convergence of measures.

(iii) If $\left\{\mu_{n}\right\}^{*} \rightarrow$ in the space of probability measures on $\mathbb{R}^{d}$, then for any $\lambda>0, E_{\mu_{n}}^{\lambda}$ $\Gamma$-converges to $E_{\mu}^{\lambda}$ w.r.t. Hausdorff convergence of sets of $\mathcal{A}$.

(iv) Consider a sequence $\left\{\mu_{n}\right\}^{*} \mu$ in the space of probability measures on $\mathbb{R}^{d}$, and for any $n$ choose $\Sigma_{n} \in \operatorname{argmin} E_{\mu_{n}}^{\lambda}$, then upon subsequence $\Sigma_{n} \stackrel{d_{\mathcal{H}}}{\rightarrow}$ for some $\Sigma \in$ $\operatorname{argmin} E_{\mu}^{\lambda}$.

(v) Given $R>0$, consider a sequence $\left\{\gamma_{n}\right\}:[0,1] \longrightarrow \overline{B(0, R)}$ of Lipschitz curves with constant-speed parameterization, satisfying $\sup \mathcal{H}^{1}\left(\gamma_{n}\right)<\infty$ and $\sup \left\|\gamma_{n}\right\|_{B V}<\infty$. Then upon subsequence there exists a Lipschitz curve $\gamma$ such that:

- $\left\{\gamma_{n}\right\} \rightarrow \gamma$ in $C^{\alpha}$ for any $\alpha \in[0,1)$, 
- $\left\{\gamma_{n}^{\prime}\right\} \rightarrow \gamma^{\prime}$ in $L^{p}$ for any $p \in[1, \infty)$,

- $\left\{\gamma_{n}^{\prime \prime}\right\} \rightarrow \gamma^{\prime \prime}$ in the space of signed finite Borel measures on $\mathbb{R}^{d}$.

We also need a basic result on the nature of path connectedness of $\Sigma$. Given points $p, q \in \Sigma$, the following terminilogy will be used:

- a "curve between $p$ and $q$ " is a continuous (not necessarily injective) mapping $\gamma$ : $[0,1] \longrightarrow \Sigma$ with $\gamma(0)=p, \gamma(1)=q$.

- a "path between $p$ and $q$ " is the image of a curve $\gamma:[0,1] \longrightarrow \Sigma$ with $\gamma(0)=p$, $\gamma(1)=q$.

Lemma 2.2. Consider $X \in \mathcal{A}$ with $\mathcal{L}^{d}(\partial X)=0$. Given distinct points $p, q \in X$ there exists a minimal (w.r.t. set inclusion) path connecting them. Moreover such path can be parameterized by an injective curve.

Proof. Consider $X \in \mathcal{A}$ and $p, q \in X$ as above. Since $X$ is pathwise connected, there exists a (continuous) mapping $\gamma:[0,1] \rightarrow X$ such that $\gamma(0)=p$ and $\gamma(1)=q$. Define the family

$$
\mathcal{F}:=\{S \subseteq X: p, q \in S, S \text { compact path }\},
$$

with the order " $\supseteq$ ". Obviously $\mathcal{F}$ in non empty as it contains $\gamma([0,1])$ in $X$, which is compact. It is easy to check that $(\mathcal{F}, \supseteq)$ satisfies hypothesis of Zorn's lemma, as every ascending chain $\left\{S_{i}\right\}_{i \in J}$ (where $J$ is a arbitrary set of indexes) has upper bound $\bigcap_{i \in J} S_{i}$, which is still an element of $\mathcal{F}$. Thus there exists a maximal element $S^{*} \in \mathcal{F}$. This $S^{*}$ can be parameterized by an injective curve: indeed given a non injective parameterization $\gamma:[0,1] \longrightarrow S^{*}$, with $\gamma(0)=p, \gamma(1)=q$, for which there exist $0<t<s<1$ such that $\gamma(t)=\gamma(s)$ and $\gamma([t, s]) \supsetneq \gamma(t)$, then $\gamma([0, t] \cup[s, 1]) \in \mathcal{F}$ would contradict the maximality of $S^{*}$ unless it has the same image $S^{*}=\gamma([0,1])$. In this latter case $\gamma([0, t] \cup[s, 1])$ can be used to parameterize $S^{*}$, and this argument can be repeated whenever $\gamma$ is not injective.

In view of this result, the expression "injective path" will be used throughout the paper to denote a path admitting an injective parameterization, while a "minimal path" between two distinct points always refers to a minimal, compact, injective path between them. Notice that nothing is claimed about uniqueness of such minimal paths. We show below that if the minimal path is not unique then the set contains a loop. Since the minimizers of $E_{\mu}^{\lambda}$ cannot contain loops, this implies that for minimizers the minimal path between two points is unique.

Lemma 2.3. Assume $X \in \mathcal{A}$ with $\mathcal{L}^{d}(\partial X)=0$ and $p, q \in X$ are distinct points. If there exist distinct minimal paths parameterized by

$$
\gamma_{1}, \gamma_{2}:[0,1] \longrightarrow X, \quad \gamma_{1}(0)=\gamma_{2}(0)=p, \gamma_{1}(1)=\gamma_{2}(1)=q,
$$

then $X$ contains a loop. 
Proof. Note that $\gamma_{1}([0,1]) \nsubseteq \gamma_{2}([0,1])$ and $\gamma_{2}([0,1]) \nsubseteq \gamma_{1}([0,1])$ since otherwise paths are either not distinct or at least one is not minimal. Denote with $L_{i}:=\gamma_{i}([0,1]), i=1,2$, and the set $L_{1} \cap L_{2}$ is compact. Using Lemma 2.2 it can be assumed $L_{1}, L_{2}$ minimal injective paths. By hypothesis neither $L_{1} \subseteq L_{2}$ nor $L_{2} \subseteq L_{1}$ holds, thus $L_{1} \backslash L_{2}$ and $L_{2} \backslash L_{1}$ are both non empty sets, open in both $L_{1}$ and $L_{2}$ (obviously, if $x \in L_{1} \backslash L_{2}, d_{\mathcal{H}}\left(\{x\}, L_{2}\right)>0$, thus there exists $\varepsilon>0$ such that $B(x, \varepsilon) \cap L_{2}=\emptyset$ and all points of $L_{1} \cap B(x, \varepsilon)$ do not belong to $L_{2}$ ).

Choose an arbitrary $x \in L_{1} \backslash L_{2}$; as $L_{1} \cap L_{2}$ is compact in $L_{1}$, the difference $L_{1} \backslash\left(L_{1} \cap L_{2}\right)$ is open in $L_{1}$. As $L_{1}$ is the continuous image of $[0,1]$ through an injective function, and $p, q \in L_{1} \cap L_{2}, x \notin\{p, q\}$. Denote with $U$ the connected component of $L_{1} \backslash L_{2}$ containing $x$. Its boundary $\partial U$ is composed by exactly 2 points $y_{1}, y_{2}$, as $U$ is connected and $\gamma_{1}$ is injective. Without loss of generality assume $\gamma_{i}:[0,1] \longrightarrow L_{i}, i=1,2$ to be constant speed parameterization, with $\gamma_{i}(0)=p, \gamma_{i}(1)=q$. Thus $\gamma_{1}(t)=x$ for some $t \in(0,1)$, and $\gamma\left(\left(t_{1}, t_{2}\right)\right)=U$ for some $t_{1}<t<t_{2}$. Injectivity implies $\gamma_{1}\left(t_{1}\right)=y_{1}$ and $\gamma_{1}\left(t_{2}\right)=y_{2}$. By construction $\gamma_{1}\left(t_{1}\right), \gamma_{1}\left(t_{2}\right) \in L_{2}$, as $U$ is a connected component of $L_{1} \backslash L_{2}$. Consequently there exist $s_{1}<s_{2} \in(0,1)$ such that $\gamma_{2}\left(s_{i}\right)=\gamma_{1}\left(t_{i}\right), i=1,2$. Both $\gamma_{1}\left(\left[t_{1}, t_{2}\right]\right)$ and $\gamma_{2}\left(\left[s_{1}, s_{2}\right]\right)$ are paths connecting those 2 points, and $\gamma_{1}\left(\left(t_{1}, t_{2}\right)\right) \cap \gamma_{2}\left(\left(s_{1}, s_{2}\right)\right)=\emptyset$ as otherwise would contradict the connectedness of $U$. Thus $\gamma_{1}\left(\left[t_{1}, t_{2}\right]\right) \cup \gamma_{2}\left(\left[s_{1}, s_{2}\right]\right)$ is a loop.

In view of Lemmas 2.2 and 2.3 , it follows that given two points $p, q \in \Sigma$ there exists a unique minimal path $A \subseteq$ between $p$ and $q$.

2.1. Discrete approximations. We start by recalling the setup and some results from [10].

Definition 2.4. Given points $y_{1}, \ldots, y_{n} \in \mathbb{R}^{d}$, a Steiner graph $S t\left(y_{1}, \ldots, y_{n}\right)$ for the points is a set of minimal length containing $y_{1}, \ldots, y_{n}$.

We note that in general Steiner graph for the given set of points in not unique. Here we list a few basic properties of Steiner graphs, their proofs and more on Steiner graphs can be found in [4] and [5].

Proposition 2.5. Let $G$ be a Steiner graph.

(i) $G$ is a tree with straight edges,

(ii) The order of any point of $G$ does not exceed 3,

(iii) If $v \in G$ has order 3, then the edges intersecting in $v$ are coplanar, forming 3 angles measuring $2 \pi / 3$ each.

The next definition is similar to the notion of curvature:

Definition 2.6. Given a graph with straight edges $\Sigma$ and a vertex $v \in \Sigma$ with degree 2, denote by $w_{1}, w_{2}$ its neighbors. The turning angle at $v$ is

$$
T A(v):=\pi-\angle w_{1} v w_{2} .
$$


The turning angle for a subset $A \subseteq \Sigma$ is defined to be the sum of all turning angles at vertices of degree 2 which belong to $A$.

The following facts were established in [10].

(i) If $\mu$ is a discrete probability measure, then for any $\lambda>0$, any $\Sigma \in \operatorname{argmin} E_{\mu}^{\lambda}$ is a Steiner graph for a set of points (which in general are not the points in the support of $\mu$ ).

(ii) Given a discrete probability measure, $\mu, \lambda>0, \Sigma \in \operatorname{argmin} E_{\mu}^{\lambda}$, then for any endpoint $v \in \Sigma$ it holds

$$
\sigma(\mu, \Sigma, v) \geq \lambda
$$

(iii) Given $\mu$ discrete probability measure, $\lambda>0, \Sigma \in \operatorname{argmin} E_{\mu}^{\lambda}$, then any $A \subseteq \Sigma$ measurable

$$
T A(A) \leq \frac{\pi}{2 \lambda} \sigma(\mu, \Sigma, A)
$$

\section{ENDPOINT ESTIMATES}

We first establish an upper bound on the number of endpoints by proving a lower bound on the mass that projects on each endpoint.

Lemma 3.1. Given a finite measure $\mu$, a parameter $\lambda>0$, and $\Sigma \in \operatorname{argmin} E_{\mu}^{\lambda}$, it holds $\sigma(\mu, \Sigma, v) \geq \lambda$ for any endpoint $v \in \Sigma$.

For discrete measures $\mu$ this is the result (2.2); here we prove it for general measures. Proof. Choose an arbitrary endpoint $v \in \Sigma$, in [2] it has been proven that there exists $r=r(v)>0$ such that $\Sigma \backslash B(v, r) \in \mathcal{A}$. Clearly

$$
d(x, \Sigma) \leq d(x, \Sigma \backslash B(v, r))+r, \quad \mathcal{H}^{1}(\Sigma \backslash B(v, r)) \leq \mathcal{H}^{1}(\Sigma)-r .
$$

Integrating the first inequality gives $F_{\mu}(\Sigma \backslash B(v, r))-F_{\mu}(\Sigma) \leq r \sigma(\mu, \Sigma, \Sigma \cap B(v, r))$. The minimality of $\Sigma$ gives

$$
\begin{aligned}
F_{\mu}+\lambda \mathcal{H}^{1}(\Sigma) & \leq F_{\mu}(\Sigma \backslash B(v, r))+\lambda \mathcal{H}^{1}(\Sigma \backslash B(v, r)) \\
& \leq F_{\mu}+r \sigma(\mu, \Sigma, \Sigma \cap B(v, r))+\lambda\left(\mathcal{H}^{1}(\Sigma)-r\right),
\end{aligned}
$$

and passing to the limit $r \rightarrow 0^{+}$gives $\sigma(\mu, \Sigma, v) \geq \lambda$.

We show that minimizers of the average-distance problem cannot contain loops. A related result was shown in [1] for $\mu$ absolutely continuous with respect to Lebesgue measure.

Lemma 3.2. Given a finite measure $\mu$, a parameter $\lambda>0$, and $\Sigma \in \operatorname{argmin} E_{\mu}^{\lambda}$, the set $\Sigma$ does not contain a loop. 
Proof. Suppose that $\Sigma$ contains a loop $E$, and let $\varphi:[0,1] \longrightarrow E$ be a constant speed parameterization, with $\varphi(0)=\varphi(1)$ and injective in $(0,1)$. Choose an arbitrary (large) $N \in \mathbb{N}$, and partition $E$ into $N$ mutually disjoint measurable sets $I_{1}, \cdots, I_{N}$, with $I_{j}:=$ $\varphi([(j-1) / N, j / N))$. Clearly $\mathcal{L}^{1}\left(I_{j}\right)=\mathcal{H}^{1}(E) / N$ for any $j$.

Denote with $\left\{C_{j}^{k}\right\}_{k \in \mathcal{J}_{j}}$ the set of connected components of $\Sigma \backslash I_{j}$ with positive length and not intersecting $E$ - with $\mathcal{J}_{j}$ a suitable set of indexes. Then choosing $N>2 / \lambda$ for at least one index $j_{0}$ it holds

$$
\sigma\left(I_{j_{0}} \cup\left\{C_{j_{0}}^{k}\right\}_{k \in \mathcal{J}_{j_{0}}}\right)<\lambda / 2
$$

Consider the competitor $\Sigma^{\prime}$ defined in the following way:

(i) remove $I_{j}$ from $\Sigma$,

(ii) for $k \in \mathcal{J}_{j_{0}}$, choose $p_{k} \in C_{j_{0}}^{k} \cap I_{j_{0}}$, and at least one such $p_{k}$ exists as $\Sigma$ is connected; noticing that $\varphi\left(j_{0} / N\right) \in \Sigma \backslash I_{j_{0}}$, denote with $T_{k}(x):=x+\left(\varphi\left(j_{0} / N\right)-p_{k}\right)$ the translation by the vector $\varphi\left(j_{0} / N\right)-p_{k}$, and replace $C_{j_{0}}^{k}$ with $T_{k}\left(C_{j_{0}}^{k}\right)$ in $\Sigma \backslash I_{j_{0}}$. Repeat this argument for any $k \in \mathcal{J}_{j_{0}}$, and denote with $\Sigma^{\prime}$ the resulting set. By construction $\Sigma^{\prime} \in \mathcal{A}$.

As $\sigma\left(I_{j}\right)<\lambda / 2$, and each $T_{k}$ is a translation by a vector $\varphi\left(j_{0} / N\right)-p_{k}$, and $\mid \varphi\left(j_{0} / N\right)-$ $p_{k} \mid \leq \mathcal{H}^{1}(E) / N$, it follows $F_{\mu}\left(\Sigma^{\prime}\right) \leq F_{\mu}(\Sigma)+\frac{\lambda}{2} \frac{\mathcal{H}^{1}(E)}{N}$, while by construction $\mathcal{H}^{1}\left(\Sigma^{\prime}\right) \leq$ $\mathcal{H}^{1}(\Sigma)-\mathcal{H}^{1}(E) / N$, contradicting the minimality of $\Sigma$.

Lemma 3.3. Given a discrete measure $\mu$ a minimizer $X \in \operatorname{argmin} E_{\mu}^{\lambda}$ with $\mathcal{H}^{1}(X)>0$, and a point $p \in X$, then each connected component of $X \backslash\{p\}$ must contain an endpoint of $X$.

Proof. First observe that $X \backslash\{p\}$ cannot be empty. Consider the family

$$
\mathcal{F}:=\{S \subseteq X: S \text { simple curve with one endpoint at } p\}
$$

ordered by inclusion.

This family cannot be empty: indeed as $X \ni p$, given another point $X \ni q \neq p$ the minimal path $\gamma \subseteq X$ connecting $q$ and $p$, which is obtained as the intersection of all paths connecting $q$ and $p$, is a simple curve. The family $\mathcal{F}$ satisfies conditions of Zorn's lemma: indeed for any ascending chain $\left\{S_{i}\right\}_{i \in J}$, the union $\bigcup_{i \in J} S_{i}$ is an upper bound. Thus there exists a maximal element $S^{*}$, which is still a simple curve, compact (if this is not the case one can consider the closure $S^{*}$, which is still an element of $\mathcal{F}$ ), with one endpoint in $P$, and the contains another endpoint: indeed choose a point $q \in S^{*}$ such that $d_{S^{*}}(p, q)$ is maximal, with $d_{S^{*}}$ denoting the path distance on $S^{*}$, and if $q$ has order at least 2, then (using Menger $n$-Beinsatz) there exists another simple curve $S^{\prime}$ with endpoint in $q$, disjoint from $S^{*}$ in a neighborhood of $q$, and $S^{*} \cup S^{\prime}$ contradicts the maximality of $S^{*}$. 
The main result of this section deals with the relation of endpoints of minimizers corresponding to discrete approximation of $\mu$ and the endpoints of a minimizer, $\Sigma$, corresponding to $\mu$. Recall that by $\operatorname{ext}(\Sigma)$ we denote the set of endpoints of $\Sigma$.

Theorem 3.4. Let $\mu$ be a compactly supported probability measure. Given a sequence of probability measures $\left\{\mu_{n}\right\} \stackrel{*}{\rightarrow} \mu$, with uniformly bounded support, for any $n$ choose an element

$$
\Sigma_{n} \in \operatorname{argmin} E_{\mu_{n}}^{\lambda} .
$$

Then upon subsequence $\left\{\Sigma_{n}\right\} \stackrel{d_{\mathcal{H}}}{\rightarrow} \Sigma$ for some $\Sigma \in \operatorname{argmin} E_{\mu}^{\lambda}$.

$B y$ relabeling the indices we can assume that the subsequence is the whole sequence. Then for any endpoint $z \in \operatorname{ext}(\Sigma)$ there exists a sequence of endpoints $z_{n} \in \exp \left(\Sigma_{n}\right)$ such that $z_{n} \rightarrow z$ as $n \rightarrow \infty$. This in particular implies

$$
\liminf _{n \rightarrow \infty} \sharp \operatorname{ext}\left(\Sigma_{n}\right) \geq \sharp \operatorname{ext}(\Sigma) .
$$

This estimate is crucial in the next section, when we discuss the topological relation between minimizers of $E_{\mu}^{\lambda}$ and minimizers of $E_{\mu_{n}}^{\lambda}$, where $\mu_{n}$ is a discrete approximation to $\mu$.

Proof. By our assumptions there exists $R>0$ such that for all $n$, supp $\mu_{n} \subseteq \bar{B}(0, R)$. Note that then $\Sigma_{n} \subseteq \bar{B}(0, R)$. Let us also note that by (2.1) the lengths $\mathcal{H}^{1}\left(\Sigma_{n}\right)$ are uniformly bounded. By Blaschke's theorem, along a subsequence $\Sigma_{n} \stackrel{d_{\mathcal{H}}}{\rightarrow}$ as $n \rightarrow \infty$. By relabeling the subsequence we can assume that it is the whole sequence. The lowersemicontinuity of the $\mathcal{H}^{1}$ with respect to Hausdorff metric proved by Gołab and the continuity of $F_{\mu}(\Sigma)$ in both $\mu$ (with respect to weak-* topology) and $\Sigma$ (with respect to Hasudorff metric) implies that $\Sigma$ is a minimizer of $E_{\mu}^{\lambda}$. Furthermore for any endpoint $z$ of $\Sigma$ the convergence $\Sigma_{n}$ to $\Sigma$ in Hausdorff metric implies that there exists a sequence $z_{n} \in \Sigma_{n}$ such that $z_{n} \rightarrow z$ as $n \rightarrow \infty$.

If $z_{n}$ are all endpoints then there is nothing to prove. We start the discussion by assuming that $z_{n}$ has a subsequence of points of order 2 (triple junctions are considered later). By relabeling we can assume that all of $z_{n} \in \Sigma_{n}$ are of order 2 . We denote by $\Sigma_{n}^{\prime}$ and $\Sigma_{n}^{\prime \prime}$ the two connected components of $\Sigma_{n} \backslash\left\{z_{n}\right\}$. We also choose sequences $\left\{v_{n}\right\},\left\{x_{n}\right\}$ both converging to $z$ and such that $v_{n} \in \Sigma_{n}^{\prime}, x_{n} \in \Sigma_{n}^{\prime \prime}$ for any $n$.

For set $X \subset \mathbb{R}^{d}$ let $r(X)=\sup _{y \in X}|y-z|$. The following dichotomy applies:

$(\sharp) \limsup _{n \rightarrow \infty} \min \left\{r\left(\Sigma_{n}^{\prime}\right), r\left(\Sigma_{n}^{\prime \prime}\right)\right\}=0$

(*) There exists $\beta>0$ and a subsequence $\left\{n_{k}\right\}_{k=1,2, \ldots}$ such that for all $k$ large enough $\min \left\{r\left(\Sigma_{n_{k}}^{\prime}\right), r\left(\Sigma_{n_{k}}^{\prime \prime}\right)\right\} \geq \beta$.

If $(\sharp)$ holds then, since by Lemma 3.3 both $\Sigma_{n}^{\prime}$ and $\Sigma_{n}^{\prime \prime}$ contain at least one endpoint, there exists an endpoint, $\tilde{z}_{n}$, at distance at most $\min \left\{r\left(\Sigma^{\prime}\right), r\left(\Sigma^{\prime \prime}\right)\right\}$ to $z_{n}$. Then $\tilde{z}_{n} \rightarrow z$ as $n \rightarrow \infty$ and thus $z$ is still a limit of endpoints as desired. 


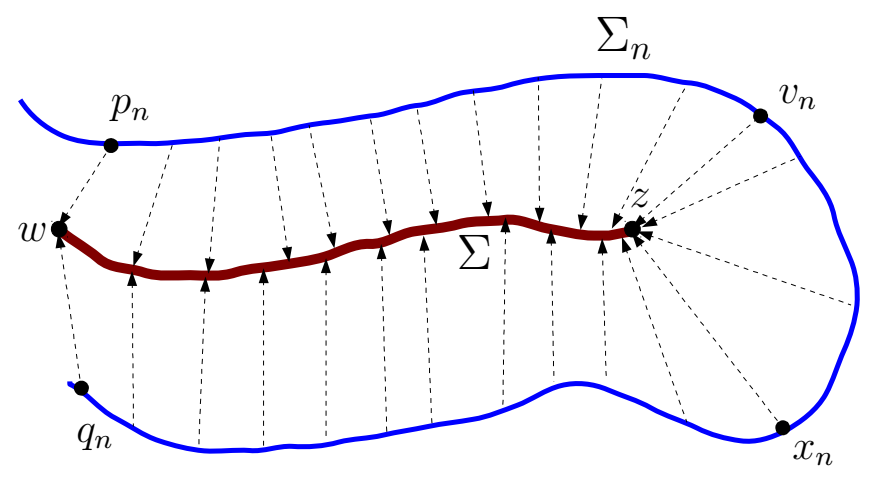

FIGURE 1. $\Sigma$ is an example of double line.

What remains is to show that the case $(*)$ is impossible. This requires a detailed geometric argument which we divide in several parts. If $(*)$ holds then, from Blaschke's compactness theorem, follows that along a further subsequence, which we relabel to be the whole sequence, both connected components converge in $d_{\mathcal{H}}$, to sets with positive length. More precisely

$$
\Sigma^{\prime}:=\lim _{n \rightarrow \infty} \Sigma_{n}^{\prime} \cup\left\{z_{n}\right\}, \quad \Sigma^{\prime \prime}:=\lim _{n \rightarrow \infty} \Sigma_{n}^{\prime \prime} \cup\left\{z_{n}\right\}
$$

We observe that $z \in \Sigma^{\prime} \cup \Sigma^{\prime \prime}$ and that $r\left(\Sigma^{\prime}\right) \geq \beta$ and $r\left(\Sigma^{\prime \prime}\right) \geq \beta$. Therefore $\mathcal{H}^{1}\left(\Sigma^{\prime}\right) \geq \beta$ and $\mathcal{H}^{1}\left(\Sigma^{\prime \prime}\right) \geq \beta$.

We claim that there exists a point besides $z$ in $\Sigma^{\prime} \cap \Sigma^{\prime \prime}$. The reason is that, if $\Sigma^{\prime} \cap \Sigma^{\prime \prime}=\{z\}$ then $z$ could not be an endpoint, because as $\Sigma$ does not contain loops, $\Sigma \backslash\{z\}$ cannot be connected. Thus $\Sigma^{\prime}$ and $\Sigma^{\prime \prime}$ are connected components and so $z$ is not of order one. So let $w \in \Sigma^{\prime} \cap \Sigma^{\prime \prime} \backslash\{z\}$. Denote by $\Sigma_{z, w}^{\prime}, \Sigma_{z, w}^{\prime \prime}$ two arbitrary minimal paths connecting $z$ and $w$ in $\Sigma^{\prime}$ and $\Sigma^{\prime \prime}$ respectively. As $\Sigma_{z, w}^{\prime} \neq \Sigma_{z, w}^{\prime \prime}$ would imply the existence of a loop (in view of Lemma 2.3), $\Sigma_{z, w}^{\prime}=\Sigma_{z, w}^{\prime \prime}$ must hold. Define $\alpha:=\mathcal{H}^{1}\left(\Sigma_{z, w}^{\prime}\right)$.

It is possible to choose sequences $\left\{p_{n}\right\},\left\{q_{n}\right\}$ with $p_{n} \in \Sigma_{n}^{\prime}, q_{n} \in \Sigma_{n}^{\prime \prime}$, both converging to $w$, and such that $\min \left\{\left|p_{n}-v_{n}\right|,\left|q_{n}-x_{n}\right|\right\} \geq|z-w| / 2$ for any $n$. Notice that in all this argument the condition " $z$ is an endpoint of $\Sigma$ " implies that $z$ is an endpoint for any subset of $\Sigma$ containing it, including $\Sigma^{\prime}, \Sigma^{\prime \prime}, \Sigma_{z, w}^{\prime}$, and $\Sigma_{z, w}^{\prime \prime}$.

Definition 3.5. If $\Sigma_{n} \rightarrow \Sigma$, a closed subset $L \subseteq \Sigma$ is a "double line" if it is a minimal path connecting distinct points $v$ and $w$ (in $\Sigma$ ) and there exist points $\left\{v_{n}, x_{n}, p_{n}, q_{n}\right\} \subseteq \Sigma_{n}$ (where $v_{n}$ may be equal to $\left.x_{n}\right)$, satisfying

- $v_{n} \rightarrow v, x_{n} \rightarrow v, p_{n} \rightarrow w, q_{n} \rightarrow$ as $n \rightarrow \infty$, 
- the minimal path between $p_{n}$ and $q_{n}$ contains $\left\{v_{n}, x_{n}\right\}$ for any $n$.

It can be shown that, since $\Sigma$ does not contain any loops, that if $\Sigma$ has a double line, then it has a double line such that $v$ is an endpoint. However we do not need this fact in our arguments.

Notice that construction in case $(*)$ implies the presence of a double line in the limit set $\Sigma$.

Lemma 3.6. Given a sequence of discrete probability measures $\left\{\mu_{n}\right\}$ converging to some probability measure $\mu$ w.r.t. weak-* topology, and a sequence of minimizers $\Sigma_{n} \in \operatorname{argmin} E_{\mu_{n}}^{\lambda}$ converging to $\Sigma \in \operatorname{argmin} E_{\mu}^{\lambda}$ w.r.t. $d_{\mathcal{H}}$, then the set $\Sigma$ cannot contain double lines.

Proof. The proof is done by contradiction: assume there exists a double line $L \subseteq \Sigma$. The aim is to find a competitor $\tilde{\Sigma}_{n}$ contradicting the optimality of $\Sigma_{n}$.

From the definition of a double line there exists distinct points $v, w \in L$ and distinct points $\left\{v_{n}, x_{n}, p_{n}, q_{n}\right\} \subseteq \Sigma_{n}$ for all $n$, satisfying

$$
v_{n} \rightarrow v, x_{n} \rightarrow v, p_{n} \rightarrow w, q_{n} \rightarrow w \text { as } n \rightarrow \infty .
$$

From the discussion above it is possible to choose these sequences such that there exist disjoint curves $\gamma_{n}^{\prime}, \gamma_{n}^{\prime \prime}:[0,1] \longrightarrow \Sigma_{n}$ connecting $p_{n}$ to $v_{n}$, and $q_{n}$ to $x_{n}$ respectively, and their respective image $L_{n}^{\prime}, L_{n}^{\prime \prime}$ both converge to $L$ w.r.t. $d_{\mathcal{H}}$. Let $L_{n}:=L_{n}^{\prime} \cup L_{n}^{\prime \prime}$. It also holds that $\Sigma_{n} \backslash L_{n} \cup L \rightarrow \Sigma$ w.r.t. $d_{\mathcal{H}}$ as $n \rightarrow \infty$. By lower semicontinuity of $\mathcal{H}^{1}$ :

$$
\liminf _{n \rightarrow \infty} \min \left\{\mathcal{H}^{1}\left(L_{n}^{\prime}\right), \mathcal{H}^{1}\left(L_{n}^{\prime \prime}\right)\right\} \geq \mathcal{H}^{1}(L) \geq d_{L}(v, w)=: a>0,
$$

where $d_{L}$ denotes the path distance on $L$. It follows that $\liminf _{n \rightarrow \infty} \mathcal{H}^{1}\left(L_{n}\right) \geq 2 \mathcal{H}^{1}(L) \geq$ $2 a$, i.e. for $n$ sufficiently large $\mathcal{H}^{1}\left(L_{n}\right) \geq 1.9 \mathcal{H}^{1}(L)$ holds.

Choose $\varepsilon>0$, and $n$ sufficiently large such that

$$
\max \left\{d_{\mathcal{H}}\left(\Sigma_{n}, \Sigma\right), d_{\mathcal{H}}\left(L_{n}, L\right), d_{\mathcal{H}}\left(\Sigma_{n} \backslash L_{n} \cup L, \Sigma\right),\left|p_{n}-w\right|,\left|q_{n}-w\right|,\left|v_{n}-v\right|\right\}<\varepsilon .
$$

Denote with $[x, y]$ the line segment with endpoints in $x$ and $y$. Define

$$
A_{n}:=\left(\Sigma_{n} \backslash L_{n}\right) \cup\left[p_{n}, w\right] \cup\left[q_{n}, w\right] \cup\left[v_{n}, v\right] \cup\left[x_{n}, v\right] \cup L,
$$

i.e. $A_{n}$ is obtained from $\Sigma_{n}$ by first removing $L_{n}$ and then replacing it with line segments $\left[p_{n}, w\right],\left[q_{n}, w\right]\left[v_{n}, v\right],\left[x_{n}, v\right]$ and $L$. For any sets $A, B \subset \mathbb{R}^{d}$ we define

$$
d_{a \mathcal{H}}(A, B)=\sup _{b \in B} \inf _{a \in A}|a-b| .
$$

Note that $d_{\mathcal{H}}(A, B)=\max \left\{d_{a \mathcal{H}}(A, B), d_{a \mathcal{H}}(B, A)\right\}$. Then $d_{a \mathcal{H}}\left(\Sigma_{n},\left[p_{n}, w\right]\right) \leq\left|p_{n}-w\right|<\varepsilon$, and similarly $d_{a \mathcal{H}}\left(\Sigma_{n},\left[q_{n}, w\right]\right)<\varepsilon, d_{a \mathcal{H}}\left(\Sigma_{n},\left[v_{n}, v\right]\right)<\varepsilon, d_{a \mathcal{H}}\left(\Sigma_{n},\left[x_{n}, v\right]\right)<\varepsilon$. Combining 
with $d_{\mathcal{H}}\left(\Sigma_{n} \backslash L_{n} \cup L, \Sigma\right)<\varepsilon$ gives $d_{a \mathcal{H}}\left(A_{n}, \Sigma_{n}\right)<\varepsilon$. Moreover it holds

$$
\begin{aligned}
\mathcal{H}^{1}\left(A_{n}\right) & \leq \mathcal{H}^{1}\left(\Sigma_{n}\right)-\mathcal{H}^{1}\left(L_{n}\right)+\left|p_{n}-w\right|+\left|q_{n}-w\right|+\left|v_{n}, v\right|+\left[x_{n}, v\right]+\mathcal{H}^{1}(L) \\
& \leq \mathcal{H}^{1}\left(\Sigma_{n}\right)-\mathcal{H}^{1}\left(L_{n}\right)+\mathcal{H}^{1}(L)+4 \varepsilon \\
& \leq \mathcal{H}^{1}\left(\Sigma_{n}\right)-0.9 \mathcal{H}^{1}(L)+3 \varepsilon \\
& \leq \mathcal{H}^{1}\left(\Sigma_{n}\right)-0.9 a+3 \varepsilon .
\end{aligned}
$$

The issue we still face is that $A_{n}$ may not belong to $\mathcal{A}$. Namely $A_{n}$ may be disconnected (for example if $L_{n}$ contains triple junctions). Let $C_{0}^{n}$ be the connected component of $A_{n}$ containing $L$. Let $\mathcal{F}^{n}:=\left\{C_{j}^{n}\right\}_{j \in I}$ be the family of connected components of $A_{n}$, other than $C_{0}^{n}$. Since each connected component must contain an endpoint of $\Sigma_{n}$, by Lemma 3.1. $I$ must be finite. We now translate these components so that they connect to $L$. Consider an arbitrary $C_{j}^{n} \in \mathcal{F}^{n}$. As $L_{n}$ is the only set present in $\Sigma_{n}$ but not present in $A_{n}$, there exists a point $s_{j}^{n} \in L_{n} \cap C_{j}^{n}$. Define

$$
T_{\theta}: \mathbb{R}^{d} \longrightarrow \mathbb{R}^{d}, \quad T_{\theta}(x):=x+\theta
$$

the translation by a vector $\theta$, and let $\pi_{L}$ be the projection on $L$, i.e. for any $x$ the point $\pi_{L}(x)$ satisfies $\left|x-\pi_{L}(x)\right|=d(x, L)$ (if there is more than one minimizer, then $\pi_{L}(x)$ can be chosen arbitrarily among these). Define

$$
\tilde{\Sigma}_{n}:=\left(A_{n} \backslash \bigcup_{j \in I} C_{j}^{n}\right) \cup \bigcup_{j \in I} T_{\pi_{L}\left(s_{j}^{n}\right)-s_{j}^{n}}\left(C_{j}^{n}\right)
$$

It is pathwise connected and compact by construction. Notice that $d_{\mathcal{H}}\left(L_{n}, L\right)<\varepsilon$ implies $\left|s_{j}^{n}-\pi_{L}\left(s_{j}^{n}\right)\right|<\varepsilon$. Therefore $d_{a \mathcal{H}}\left(\tilde{\Sigma}_{n}, A_{n}\right) \leq \varepsilon$, which combined with $d_{a \mathcal{H}}\left(A_{n}, \Sigma_{n}\right)<\varepsilon$ gives $d_{a \mathcal{H}}\left(\tilde{\Sigma}_{n}, \Sigma_{n}\right) \leq 2 \varepsilon$. From $\left|d\left(x, \tilde{\Sigma}_{n}\right)-d\left(x, \Sigma_{n}\right)\right| \leq d_{a \mathcal{H}}\left(\tilde{\Sigma}_{n}, \Sigma_{n}\right) \leq 2 \varepsilon$, integrating on $\mathbb{R}^{d}$ yields

$$
\left|\int d\left(x, \tilde{\Sigma}_{n}\right) d \mu_{n}-\int d\left(x, \Sigma_{n}\right) d \mu_{n}\right| \leq \mu_{n}\left(\mathbb{R}^{d}\right) d_{\mathcal{H}}\left(\tilde{\Sigma}_{n}, \Sigma_{n}\right) \leq 2 \varepsilon .
$$

The last step involves estimating $\mathcal{H}^{1}\left(\tilde{\Sigma}_{n}\right)$ : by construction $\tilde{\Sigma}_{n}$ is obtained from $A_{n}$ by first removing $\bigcup_{j \in I} C_{j}^{n}$, then adding $\bigcup_{j \in I} T_{\pi_{L}\left(s_{j}^{n}\right)-s_{j}^{n}}\left(C_{j}^{n}\right)$. Since by definition $C_{j}^{n} \cap C_{s}^{n}=\emptyset$ if $j \neq s$ and $\bigcup_{j \in I} C_{j}^{n} \subseteq \Sigma_{n}$

$$
\mathcal{H}^{1}\left(\bigcup_{j \in I} C_{j}^{n}\right)=\sum_{j \in I} \mathcal{H}^{1}\left(C_{j}^{n}\right) \leq \mathcal{H}^{1}\left(\Sigma_{n}\right)<\infty
$$


Analogously, considering that $T_{\pi_{L}\left(s_{j}^{n}\right)-s_{j}^{n}}\left(C_{j}^{n}\right)$ is the image of $C_{j}^{n}$ through a translation, $\mathcal{H}^{1}\left(T_{\pi_{L}\left(s_{j}^{n}\right)-s_{j}^{n}}\left(C_{j}^{n}\right)\right)=\mathcal{H}^{1}\left(C_{j}^{n}\right)$ for any $j$, thus

$$
\mathcal{H}^{1}\left(\bigcup_{j \in I} T_{\pi_{L}\left(s_{j}^{n}\right)-s_{j}^{n}}\left(C_{j}^{n}\right)\right)=\mathcal{H}^{1}\left(\bigcup_{j \in I} C_{j}^{n}\right)=\sum_{j \in I} \mathcal{H}^{1}\left(C_{j}^{n}\right) \leq \mathcal{H}^{1}\left(\Sigma_{n}\right)<\infty .
$$

Using (3.1) this gives

$$
\mathcal{H}^{1}\left(\tilde{\Sigma}_{n}\right)=\mathcal{H}^{1}\left(A_{n}\right) \leq \mathcal{H}^{1}\left(\Sigma_{n}\right)-0.9 a+3 \varepsilon .
$$

Combining with (3.2) we conclude

$$
E_{\mu_{n}}^{\lambda}\left(\tilde{\Sigma}_{n}\right) \leq E_{\mu_{n}}^{\lambda}\left(\Sigma_{n}\right)+2 \varepsilon-0.9 \lambda a+3 \varepsilon \lambda
$$

which for $\varepsilon$ sufficiently small contradicts the minimality of $\Sigma_{n}$.

We return to the proof of Theorem 3.4. It remains to consider the case that an endpoint $z \in \operatorname{ext}(\Sigma)$ is a limit of points $z_{n} \in \Sigma_{n}$ of order 3 . We note that arbitrarily close to any point of order 3 there exists a point of order 2 . Thus $z$ can be obtained as a limit of points of order 2, which is the case considered above.

\section{TOPOLOGICAL "LOWER SEMICONTINUITY"}

4.1. Topological relation. Given $\lambda>0$ and a compactly supported probability measure $\mu$, consider a sequence of discrete probability measures $\left\{\mu_{n}\right\} \stackrel{*}{\sim} \mu$, and for any $n$ choose a $\operatorname{minimizer} \Sigma_{n} \in \operatorname{argmin} E_{\mu_{n}}^{\lambda}$. Then upon subsequence $\Sigma_{n} \stackrel{d_{\mathcal{H}}}{\rightarrow} \in \in \operatorname{argmin} E_{\mu}^{\lambda}$. The aim of the this section is to analyze topological relations between $\Sigma_{n}$ (for $n$ sufficiently large) and $\Sigma$.

The main result is:

Theorem 4.1. Given $\lambda>0$ and a compactly supported probability measure $\mu$, consider a sequence of discrete probability measures $\left\{\mu_{n}\right\}^{*} \mu$, and for any $n$ choose a minimizer $\Sigma_{n} \in$ $\operatorname{argmin} E_{\mu_{n}}^{\lambda}$. Then, upon a subsequence, $\Sigma_{n} \stackrel{d_{\mathcal{H}}}{\rightarrow} \in \operatorname{argmin} E_{\mu}^{\lambda}$. Then then for all sufficiently large $n$ along the subsequence there exists a homeomorphism $\varphi_{n}: \Sigma \longrightarrow S_{n} \subseteq \Sigma_{n}$, for some subset $S_{n} \subseteq \Sigma_{n}$.

Proof. The convergence of $\Sigma_{n}$ along a subsequence follows from Theorem 3.4 . We again assume that the subsequence is the whole sequence. Let $V$ be the set of all endpoints and triple junctions of $\Sigma$. By Theorem 3.4. $\Sigma$ and $\Sigma_{n}$ contain at most $1 / \lambda$ endpoints. By induction on the number of triple junctions, it is easy to prove that in any tree the number of endpoints is greater than the number of triple junctions. Thus the number of 


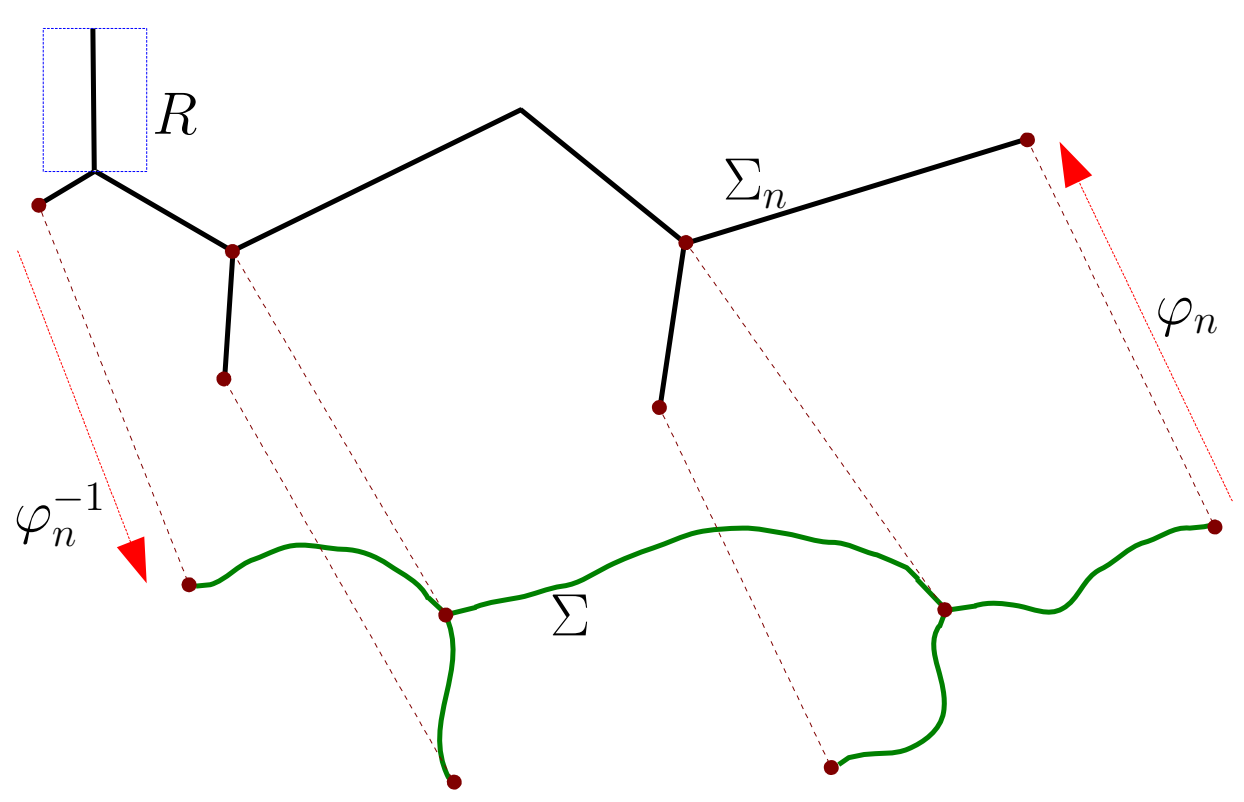

FIGURE 2. $\varphi_{n}$ is an example of homeomorphism between $\Sigma$ and a (proper) subset of $\Sigma_{n}$. The part within the dashed rectangle $R$ is not involved in the homeomorphism.

triple junctions is also bounded by $1 / \lambda$. Hence $V$ is a finite set. There exists $c>0$ such that

$$
c \leq \min _{v, \tilde{v} \in V, v \neq \tilde{v}}|v-\tilde{v}| .
$$

Choose $n$ sufficiently large such that $d_{\mathcal{H}}\left(\Sigma_{n}, \Sigma\right)<c / 2$. From Theorem 3.4 it follows that any endpoint $v$ of $\Sigma$ is limit of a sequence of endpoints $\left\{v_{n}\right\}$ of $\Sigma_{n}$. Such a sequence may be nonunique; we fix one for each endpoint of $\Sigma$. By relabeling the sequence, it can be assumed that for all endpoints $v$ of $\Sigma$ and corresponding sequence of endpoints $v_{n}$ of $\Sigma_{n}$, and all $n,\left|v-v_{n}\right|<c / 2$.

Lemma 4.2. Let $\Sigma_{n}$ and $\Sigma$ be as above. If some sequences $\left\{y_{n}\right\}$ and $\left\{w_{n}\right\}$ in $\Sigma_{n}$ converge to distinct points $y, w \in \Sigma$ then the sequence of minimal paths $\left[y_{n}, w_{n}\right]_{\Sigma_{n}}$ converges in $d_{\mathcal{H}}$ to the minimal path $[y, w]_{\Sigma}$.

Here $[y, w]_{\Sigma}$ is the minimal path in $\Sigma$ containing $y$ and $w$. The existence of such minimal path is ensured by Lemma 2.2.

Proof. Assume that this is not the case. Then there exists $\varepsilon>0$ and a subsequence of $\left[y_{n}, w_{n}\right]_{\Sigma_{n}}$ such that all paths in the subsequence are at distance at least $\varepsilon$ from $[y, w]_{\Sigma}$. By 
relabeling we can assume that this is the whole sequence. To obtain contradiction it is enough to find a (further) subsequence which does converge to $[y, w]_{\Sigma}$. By compactness we know $\left[y_{n}, w_{n}\right]_{\Sigma_{n}}$ converges along a subsequence to some connected set $A \subset \Sigma$ which contains $y$ and $w$. Since $\Sigma$ is a tree, $[y, w]_{\Sigma} \subseteq A$. Let us assume that $L=A \backslash[y, w]_{\Sigma} \neq \emptyset$. Then there exists a sequence $x_{n} \in\left[y_{n}, w_{n}\right]_{\Sigma_{n}}$ such that $x_{n} \rightarrow x \in L$ as $n \rightarrow \infty$. Let $L^{\prime}$ be the connected component of $L$ containing $x$. If $\overline{L^{\prime}} \cap[y, w]_{\Sigma}$ has two or more points then $\Sigma$ contains a loop, which contradicts the fact that $\Sigma$ is a tree. Hence $\overline{L^{\prime}} \cap[y, w]_{\Sigma}$ is a single point, denote it by $p$. Then $p \in[y, x]_{\Sigma}$ and $p \in[x, w]_{\Sigma}$. Thus there exist sequences $p_{n} \in\left[y_{n}, x_{n}\right]_{\Sigma_{n}}$ and $q_{n} \in\left[x_{n}, w_{n}\right]_{\Sigma_{n}}$ such that $p_{n} \rightarrow p$ and $q_{n} \rightarrow p$ as $n \rightarrow \infty$. Thus $\Sigma$ contains a double line which contradicts Lemma 3.6.

We return to the proof of the theorem.

Claim 1. Any triple junction $z \in \Sigma$ can be obtained as limit of a sequence of triple junctions $z_{n} \in \Sigma_{n}$. If this is not the case, there exists a triple junction $z \in \Sigma$ which cannot be obtained as limit of triple junctions. Then there exists $\varepsilon>0$ such that no point in $\Sigma \cap B(z, \varepsilon)$ is a limit of a sequence of triple junctions. By making $\varepsilon>0$ smaller we can ensure that $\Sigma \cap B(z, \varepsilon) \backslash\{z\}$ has exactly three connected components. Let $w^{1}$, $w^{2}$, and $w^{3}$ be elements of these connected components. Then there exist sequences $w_{n}^{i} \in \Sigma_{n}$ for $i=1,2,3$ such that $w_{n}^{i} \rightarrow w^{i}$ as $n \rightarrow \infty$. By Lemma 4.2 . for $i, j \in\{1,2,3\}$ distinct, $\left[w_{n}^{i}, w_{n}^{j}\right]_{\Sigma_{n}} \rightarrow\left[w^{i}, w^{j}\right]_{\Sigma}$ as $n \rightarrow \infty$. Since $\left[w^{i}, w^{j}\right]_{\Sigma} \subset B(z, \varepsilon)$, none of $\left[w_{n}^{i}, w_{n}^{j}\right]_{\Sigma_{n}}$ contains a triple junction (for $n$ large enough). Hence one of the points has to lie on the minimal path connecting the other two, say $w_{n}^{2} \in\left[w_{n}^{1}, w_{n}^{3}\right]_{\Sigma_{n}}$ for all $n$ large enough (along a subsequence). Thus $w^{2} \in\left[w^{1}, w^{3}\right]_{\Sigma}$, which contradicts the fact that all three the points lie in different connected components of $\Sigma \cap B(z, \varepsilon) \backslash\{z\}$.

Similarly to the argument made for endpoints, we can assume that for any triple junction $z \in \Sigma$, is a limit of a sequence of triple junctions $\left\{z_{n}\right\}$ such that $\left|z-z_{n}\right|<$ $c / 2$ for all $n$. This sequence may be nonunique, but we select one. From (4.1) follows that sequences converging to distinct endpoints/triple junctions have no overlapping elements. Let $V_{n}$ be the set of endpoints and triple junctions of $\Sigma_{n}$ which are in the sequences (selected above) converging to elements of $V$.

Claim 2. If $w_{1}, w_{2} \in V$ are such that $\left[w_{1}, w_{2}\right]_{\Sigma}$ does not contain endpoints/triple junctions besides $w_{1}$ and $w_{2}$ then for all $n$ large enough $\left[w_{n}^{1}, w_{n}^{2}\right]_{\Sigma_{n}}$ does not contain any elements of $V_{n}$ besides $w_{n}^{1}$ and $w_{n}^{2}$. Assume that this is not the case: that along a subsequence $V_{n} \cap\left[w_{n}^{1}, w_{n}^{2}\right]_{\Sigma_{n}}$ contains an element of $V_{n}$ other than $w_{n}^{1}$ and $w_{n}^{2}$. By considering a further subsequence we can assume that it is always from the same sequence, say $\left\{w_{n}^{3}\right\}$. That is $w_{n}^{3} \in\left[w_{n}^{1}, w_{n}^{2}\right]_{\Sigma_{n}}$ for all $n$ along a subsequence. As before we can assume that the subsequence is the whole sequence. From Lemma 4.2 it follows that $w_{3} \in\left[w^{1}, w^{2}\right]_{\Sigma}$. Contradiction. 
We are finally ready to define the desired homeomorphism. Choose a function $\varphi_{n}$ : $\Sigma \longrightarrow \varphi_{n}(\Sigma) \subseteq \Sigma_{n}$ such that:

(i) if an endpoint $v \in \Sigma$ is limit of a sequence of endpoints $\left\{v_{n}\right\}$ with $v_{n} \in V_{n}$ then $\varphi_{n}(v)=v_{n}$,

(ii) if a triple junction $z \in \Sigma$ is limit of a sequence of triple junctions $\left\{z_{n}\right\}$ with $z_{n} \in V_{n}$, then $\varphi_{n}(z)=z_{n}$,

(iii) if $w_{1}, w_{2} \in V$ are such that $\left[w_{1}, w_{2}\right]_{\Sigma}$ does not contain endpoints/triple junctions besides $w_{1}, w_{2}$, then define $\varphi_{n \mid\left[w_{1}, w_{2}\right]_{\Sigma}}$ as an arbitrary homeomorphism between $\left[w_{1}, w_{2}\right]_{\Sigma}$ and $\left[w_{n}^{1}, w_{n}^{2}\right]_{\Sigma_{n}}$, where $w_{n}^{1}, w_{n}^{2} \in V_{n}$ and $\left\{w_{n}^{1}\right\} \rightarrow w_{1},\left\{w_{n}^{2}\right\} \rightarrow w_{2}$, as $n \rightarrow \infty$.

The function $\varphi_{n}: \Sigma \rightarrow \varphi_{n}(\Sigma) \subseteq \Sigma_{n}$ is well defined, for $n$ large enough, by Claim 2 . It is injective and continuous by construction. Since $\Sigma$ is compact and $\varphi_{n}$ is a bijection, $\varphi_{n}^{-1}$ is continuous. Thus $\varphi_{n}$ is a homeomorphism.

\section{5. $B V$ ESTIMATES}

The aim of this section is to prove a regularity result about minimizers of Problem 1.1 . In [1], [2] and [3] is has been proven that any minimizer $\Sigma$ of Problem 1.1 is a countable union of Lipschitz curves. Given that, by Theorem 3.4, the total mass arriving at each endpoint is at least $\lambda$ there exists at most $1 / \lambda$ endpoints. Since the number of triple junctions in a tree is less than the number of endpoints the number of curves, forming the tree, is bounded by $1 / \lambda$. Thus $\Sigma$ is a finite union of Lipschitz curves.

The main objective of this section is to prove the following regularity result:

Theorem 5.1. Given a compactly supported finite measure $\mu$, and $\lambda>0$, any $\Sigma \in \operatorname{argmin} E_{\mu}^{\lambda}$ is finite union of Lipschitz curves $\left\{\gamma_{k}\right\}_{k=1}^{N}$ (without loss of generality assume that all $\gamma_{k}$ are arc-length parameterized), such that

$$
\sum_{k}\left\|\gamma_{k}^{\prime}\right\|_{T V} \leq \frac{1}{\lambda}\left|\mu\left(\mathbb{R}^{d}\right)\right|
$$

where $\|\cdot\|_{T V}$ denotes the total variation.

Note that we do not assume that $\mu$ is a probability measure.

The proof uses a discrete approximation of $\mu$; thus we start by proving the result for discrete measures. Given unit vectors $v_{1}$, and $v_{2}$, the "angle between" $v_{1}$ and $v_{2}$ is defined as the smallest $\alpha \geq 0$ such that

$$
\left|v_{1}-v_{2}\right|=2 \sin \frac{\alpha}{2}
$$

Lemma 5.2. Given an arbitrary discrete measure $\mu$, and $\lambda>0$, any $\Sigma \in \operatorname{argmin} E_{\mu}^{\lambda}$ is finite union of Lipschitz curves $\left\{\gamma_{k}\right\}_{k=1}^{N}$ (without loss of generality assume $\gamma_{k}$ constant speed), such 
that

$$
\left\|\gamma_{k}^{\prime}\right\|_{T V} \leq \frac{1}{\lambda} \sigma\left(\mu, \Sigma, \gamma_{k}\right)
$$

where $\sigma$ is defined after Lemma 2.1

Proof. Let $\mu$ be a probability measure. The result for general measures follows by scaling (See Section 2.1 in [10]). Consider an arbitrary minimizer $\Sigma \in \operatorname{argmin} E_{\mu}^{\lambda}$. From [10] follows that minimizers are always Steiner graphs with finitely many vertices. As Steiner graphs are trees, it follows that $\Sigma$ is finite union of arc-length parameterized Lipschitz curves $\left\{\gamma_{k}\right\}_{k=1}^{N}$, where each of these curves is union of line segments. Moreover, the number of curves, $N$, is bounded by $1 / \lambda$. Choose an arbitrary $k \in\{1, \cdots, N\}$. To simplify notations define $\gamma:=\gamma_{k}$ and $L:=\mathcal{H}^{1}(\gamma)$. Let $s_{1}<s_{2}<\cdots<s_{m}$ be the values in $[0, L]$ for which $\gamma\left(s_{i}\right)$ is a corner.

From definition of total variation and the fact that the curve is piecewise linear

$$
\left\|\gamma^{\prime}\right\|_{T V([0, L])}:=\sup _{0=t_{0}<t_{1}<\cdots<t_{M-1}<t_{M}=L} \sum_{i=0}^{M-1}\left|\gamma^{\prime}\left(t_{i+1}\right)-\gamma^{\prime}\left(t_{i}\right)\right|=\sum_{j=1}^{m}\left|\gamma^{\prime}\left(s_{j}-\right)-\gamma^{\prime}\left(s_{j}+\right)\right|
$$

where $\gamma^{\prime}(\cdot-)$ and $\gamma^{\prime}(\cdot+)$ denote the left and the right derivative respectively.

From the proof of Lemma 11 in [10] follows that

$$
\sum_{j=1}^{m}\left|\gamma^{\prime}\left(s_{j}-\right)-\gamma^{\prime}\left(s_{j}+\right)\right| \leq \frac{1}{\lambda} \sigma(\mu, \Sigma, \gamma)
$$

We should note that Lemma 11 of [10] is stated for probability measures. However it is straightforward to extend the proof to include arbitrary discrete measures, in particular in the light of the scaling presented in Section 2.1 of [10].

The inequalities above imply the desired estimate on total variation.

We remark that combining the estimate on the total variation above and the estimate (2.1) on $\mathcal{H}^{1}(\Sigma)$ we obtain an estimate on the BV norm

$$
\sum_{k=1}^{N}\left\|\gamma_{k}\right\|_{B V} \leq \frac{1}{\lambda}\left(\left|\mu\left(\mathbb{R}^{d}\right)\right|+\operatorname{diam} \operatorname{supp} \mu\right)
$$

Proof. (of Theorem 5.1) As in the proof of the lemma we assume that $\mu$ is a probability measure, as the general result follows by scaling. In [1], [2] and [3] (to which we refer for further details) it has been proven that minimizers of the average-distance functional are at most countable unions of Lipschitz curves, for the constrained formulation. It easy to notice that each minimizer $\Sigma \in \operatorname{argmin} E_{\mu}^{\lambda}$ is a minimizer of $\min _{\mathcal{H}^{1}(\mathcal{X}) \leq \mathcal{H}^{1}(\Sigma)} F_{\mu}(\mathcal{X})$ too. In Lemma 3.1 it has been proven that minimizer $\Sigma \in \operatorname{argmin} E_{\mu}^{\lambda}$ has at most $1 / \lambda$ endpoints, thus $\Sigma$ is finite union of Lipschitz curves. 
For $B V$ regularity, consider an arbitrary minimizer $\Sigma \in \operatorname{argmin} E_{\mu}^{\lambda}$. If we consider discrete approximations of $\mu$ and the corresponding minimizers $\tilde{\Sigma}_{n}$ then, as before, $\tilde{\Sigma}_{n}$ converge along a subsequence to some $\tilde{\Sigma} \in \operatorname{argmin} E_{\mu}^{\lambda}$. The problem, we need to overcome, is that $\tilde{\Sigma}$ may be different from $\Sigma$, as the minimizer is not unique in general. Note that if $\Sigma \subseteq \tilde{\Sigma}$ then there is no problem since the regularity of $\tilde{\Sigma}$ implies the regularity of $\Sigma$.

Thus we modify the measure $\mu$ in such a way that $\Sigma$ is still a minimizer of the energy corresponding to the modified measure, but that ensures that any minimizer of the energy corresponding to the modified measure contains $\Sigma$. This is one of the key ideas of the paper. In particular let $\varepsilon>0$ and

$$
\mu_{\varepsilon}:=\mu+\varepsilon \frac{1}{\mathcal{H}^{1}(\Sigma)} \mathcal{H}_{\mid \Sigma}^{1} .
$$

Note that $\Sigma$ is the minimal (w.r.t. set inclusion) minimizer of $E_{\mu_{\varepsilon}}^{\lambda}$ for any $\varepsilon>0$, i.e. every minimizer $\Sigma^{\prime} \in \operatorname{argmin} E_{\mu_{\varepsilon}}^{\lambda}$ contains $\Sigma$. Indeed, as $\Sigma$ is already a minimizer of $E_{\mu}^{\lambda}$, given an arbitrary element $\Sigma^{\prime} \in \mathcal{A}$ it holds that $E_{\mu}^{\lambda}(\Sigma) \leq E_{\mu}^{\lambda}\left(\Sigma^{\prime}\right)$. Hence if $\Sigma^{\prime}$ is a minimizer of $E_{\mu_{\varepsilon}}^{\lambda}$ then $\int_{\Sigma} d\left(x, \Sigma^{\prime}\right) d \mathcal{H}_{\mid \Sigma}^{1}=0$ and thus $\Sigma \subseteq \Sigma^{\prime}$.

Fix $\varepsilon>0$, and choose an approximating sequence $\left\{\mu_{n}\right\}{ }^{*} \mu_{\varepsilon}$, where $\mu_{n}$ is a discrete measure with $\mu_{n}\left(\mathbb{R}^{d}\right)=\mu_{\varepsilon}\left(\mathbb{R}^{d}\right)=1+\varepsilon$. For any $n$ choose $\Sigma_{n} \in \operatorname{argmin} E_{\mu_{n}}^{\lambda}$. Upon a subsequence (which we can assume to be the whole sequence), $\Sigma_{n} \stackrel{d_{\mathcal{H}}}{\rightarrow} \Sigma_{*} \in \operatorname{argmin} E_{\mu_{\varepsilon}}^{\lambda}$, which implies $\Sigma \subseteq \Sigma_{*}$.

We know that $\Sigma_{*}$ is a union of Lipschitz curves $\left\{\gamma_{*, i}: i=1, \ldots, N\right\}$ such that endpoints of all curves are either endpoints or triple junctions of $\Sigma_{*}$. By Lemma 4.2 and the proof of Theorem 4.1 for each $n$ large enough there exist curves, $\left\{\gamma_{i}^{n}: i=1, \ldots, N, n \geq\right.$ $\left.n_{0}\right\}$, with disjoint interiors in $\Sigma_{n}$ such that for each $i, \gamma_{i}^{n} \rightarrow \gamma_{*, i}$ in $d_{\mathcal{H}}$ as $n \rightarrow \infty$. Note that Lemma 5.2 gives

$$
\sum_{i=1}^{N}\left\|\left(\gamma_{i}^{n}\right)^{\prime}\right\|_{T V} \leq \frac{1+\varepsilon}{\lambda} .
$$

Since the $\mathcal{H}^{1}\left(\Sigma_{n}\right)$ are uniformly bounded $\sum_{i=1}^{N}\left\|\left(\gamma_{i}^{n}\right)^{\prime}\right\|_{B V}$ are also uniformly bounded. The estimates of Lemma 5.2 and fact (v) from the introduction (which relies on the fact that $B V$ is compactly embedded in $L^{1}$ ) imply that along a subsequence $\left(\gamma_{i}^{n}\right)^{\prime} \rightarrow \gamma_{*, i}^{\prime}$ in $L^{1}$ as $n \rightarrow \infty$.

Since total variation is lower semicontinuous with respect to $L^{1}$ convergence we conclude that for all $i=1, \ldots, N$

$$
\liminf _{n \rightarrow \infty}\left\|\left(\gamma_{i}^{n}\right)^{\prime}\right\|_{T V} \geq\left\|\gamma_{*, i}^{\prime}\right\|_{T V}
$$


Combining with (5.1), and using the lower-semicontinuity of $\mathcal{H}^{1}$ with respect to $L^{1}$ convergence of $\gamma_{i}^{n}$, we obtain

$$
\sum_{i=1}^{N}\left\|\left(\gamma_{*, i}\right)^{\prime}\right\|_{T V} \leq \frac{1+\varepsilon}{\lambda}
$$

Since the curves that make up $\Sigma$ are subsets of the curves that make up $\Sigma_{*}$ we conclude:

$$
\sum_{i=1}^{N}\left\|\left(\gamma_{i}\right)^{\prime}\right\|_{T V} \leq \frac{1+\varepsilon}{\lambda} .
$$

Taking $\varepsilon \rightarrow 0$ yields the desired result.

Again by combining the estimate on the total variation above and the estimate (2.1) on $\mathcal{H}^{1}(\Sigma)$ one obtains

$$
\sum_{k=1}^{N}\left\|\gamma_{k}\right\|_{B V} \leq \frac{1}{\lambda}\left(\left|\mu\left(\mathbb{R}^{d}\right)\right|+\operatorname{diam} \operatorname{supp} \mu\right)
$$

Acknowledgments. XYL acknowledges the support by ICTI. DS is grateful to NSF (grants DMS-0908415 and DMS-1211760) and FCT (grant UTA_CMU/MAT/0007/2009). Authors are thankful to the Center for Nonlinear Analysis (NSF grant DMS-0635983) for its support. The research was also supported by NSF PIRE grant OISE-0967140.

\section{REFERENCES}

[1] Buttazzo, G., Oudet, E., and Stepanov, E.: Optimal transportation problems with free Dirichlet regions , Progress in Nonlinear Diff. Equations and their Applications vol. 51, pp. 41-65, 2002

[2] Buttazzo, G. and Stepanov, E.: Minimization problems for average distance functionals, Calculus of Variations: Topics from the Mathematical Heritage of Ennio De Giorgi, D. Pallara (ed.), Quaderni di Matematica, Seconda Università di Napoli, Caserta vol. 14, pp. 47-83, 2004

[3] Buttazzo, G. and Stepanov, E.: Optimal transportation networks as free Dirichlet regions for the Monge-Kantorovich problem, Ann. Sc. Norm. Sup. Pisa Cl. Sci. vol. 2, pp. 631-678, 2003

[4] Gilbert, E.N. and POllack, H.O.: Steiner minimal trees, SIAM J. Appl. Math., vol 12, pp. 1-29, 1968

[5] HWANG, F.K., RICHARDS, D.S. and WINTER, P.: The Steiner tree problem, vol. 53 of Annals of Discrete Mathematics, North-Holland Publishing Co., Amsterdam, 1992

[6] LemenAnt, A.: A presentation of the average distance minimizing problem, POMI, vol. 390, pp. 117-146, 2011

[7] Lemenant, A.: About the regularity of average distance minimizers in $\mathbb{R}^{2}$, Preprint,

[8] MantegazZA, C. and MennuCCI, A.: Hamilton-jacobi equations and distance functions in Riemannian manifolds , Appl. Math. Optim., vol. 47(1), pp. 1-25, 2003

[9] PAOLINI E. and STEPANOV E.: Qualitative properties of maximum and average distance minimizers in $\mathbb{R}^{n}$, J. of Math. Sci. (N. Y.), vol. 122(3), pp. 3290-3309, 2004

[10] SLEPČEV, D.: Counterexample to regularity in average-distance problem, Preprint

(available at http://www.math.cmu.edu/users/slepcev/adp1.pdf) 
[11] Santambrogio, F. and Tilli, P.: Blow-up of Optimal Sets in the Irrigation Problem, J. Geom. Anal., vol. 15(2), pp. 343-362, 2005

[12] TILli, P.: Some explicit examples of minimizers for the irrigation problem, J. Convex Anal., vol. 17, pp. 583-595, 2010

Department of Mathematical Sciences, Carnegie Mellon University, Pittsburgh, Pa, 15213,UNITED STATES, EMAILS: XINYANG@ANDREW.CMU.EDU, SLEPCEV@MATH.CMU.EDU 